\title{
Accurate Positioning for Vehicular Safety Applications - the SAFESPOT Approach
}

\author{
Robin Schubert*, Marius Schlingelhof ${ }^{\dagger}$, Heiko Cramer* and Gerd Wanielik* \\ ${ }^{*}$ Professorship of Communications Engineering \\ Chemnitz University of Technology \\ Reichenhainer Strasse 70, 09126 Chemnitz, Germany \\ Email: \{robin.schubert;heiko.cramer;gerd.wanielik\}@etit.tu-chemnitz.de \\ Telephone: +49 37153136 475, Fax: +49 371531836475 \\ $\dagger$ Institute for Transportation Systems, German Aerospace Centre DLR \\ Rutherfordstrasse 2, 12489 Berlin, Germany \\ Email: marius.schlingelhof@dlr.de \\ Telephone: +49 3067055 290, Fax: +49 3067055202
}

\begin{abstract}
One of the main restrictions of present-day driver assistance systems is the limited temporal and spatial horizon. In order to overcome this limitation, the European integrated project SAFESPOT aims to develop a safety margin assistant, which provides the driver with appropriate recommendations for how to avoid critical situations. For reaching that goal, positioning algorithms with sub-meter accuracy are necessary. In this paper, the SAFESPOT approach for accurate relative positioning of vehicles is presented. The main idea is to combine several sources of information from a cooperative vehicle ad-hoc networks using a data fusion module. Thus, the single positioning technologies (e. g. satellite navigation, communication signals, and landmarks) as well as the data fusion algorithms are explained. The results are expected to improve localization accuracy in a way which makes it possible for vehicular safety applications to determine even the lane in which a vehicle is travelling.
\end{abstract}

\section{INTRODUCTION}

In recent years, extensive research has been carried out to develop driver assistance systems which are able to perceive potentially dangerous situations and provide the driver with an appropriate warning. However, certain problems such as reliability or temporal and spatial horizon still remain unsolved. Thus, the European integrated project SAFESPOT aims to examine how intelligent vehicles and intelligent roads can cooperate in order to overcome those restrictions. SAFESPOT, which started its activities in February 2006, is co-funded by the European Commission Information Society Technologies and includes 50 partners from 12 European countries [1].

For achieving a breakthrough in road safety with cooperative systems, innovative technologies, which are being developed in the SINTECH subproject of SAFESPOT, are necessary. The three major technical challenges include: a) The definition of reliable, fast, secure, and low-cost protocols for local vehicleto-vehicle (V2V) and vehicle-to-infrastructure (V2I) communication. b) The development of real-time relative positioning algorithms which are able to achieve sub-meter accuracy. c) The realization of real-time updateable local dynamic maps. In this paper, the SAFESPOT approach for accurate relative positioning is presented.

\section{BASIC ARCHITECTURE}

The aim of the SINTECH subproject is to develop a positioning module which is able to deliver an accurate position of the ego-vehicle in whatever environment (highway, rural, urban, et cetera) it may travel. However, no current positioning technology is able to comply with these requirements. For instance, GNSS-based systems may fail in urban environments due to multipath phenomena, whereas landmark based approaches require a sufficient number of landmarks in the vicinity of the vehicle.

In order to overcome those restrictions, the SINTECH positioning module combines several technologies, whereas most of these technologies themselves also contain new approaches or improvements of known algorithms. In figure 1, the general structure of the positioning module is illustrated. The different input systems can be divided into GNSS-based positioning, landmark-based positioning and communicationbased positioning relative to other vehicles which are travelling in the vicinity of the ego-vehicle and are connected within a vehicular ad-hoc network.

In the following, the different positioning technologies which are used within SINTECH will be briefly explained. For two approaches - the positioning based on GNSS-systems and image landmarks, respectively - a more detailed description will be given.

\section{A. GPS-based Vehicle Positioning}

Current systems today use onboard sensors that scan the vicinity up to the next obstacle. To detect another object, a line-of-sight connection is needed from the sensor to the object. For future safety applications as adressed in SAFESPOT the visible range of the onboard sensors (electronic ADAS horizon) has to be extended to look beyond the vehicles and obstacles of the direct neighbourhood. To increase this horizon and to measure the relative positions between vehicles, the navigation signals of satellites can be used. Satellite technology is primarily used for absolute positioning within a globally defined earth related coordinate system. In this 


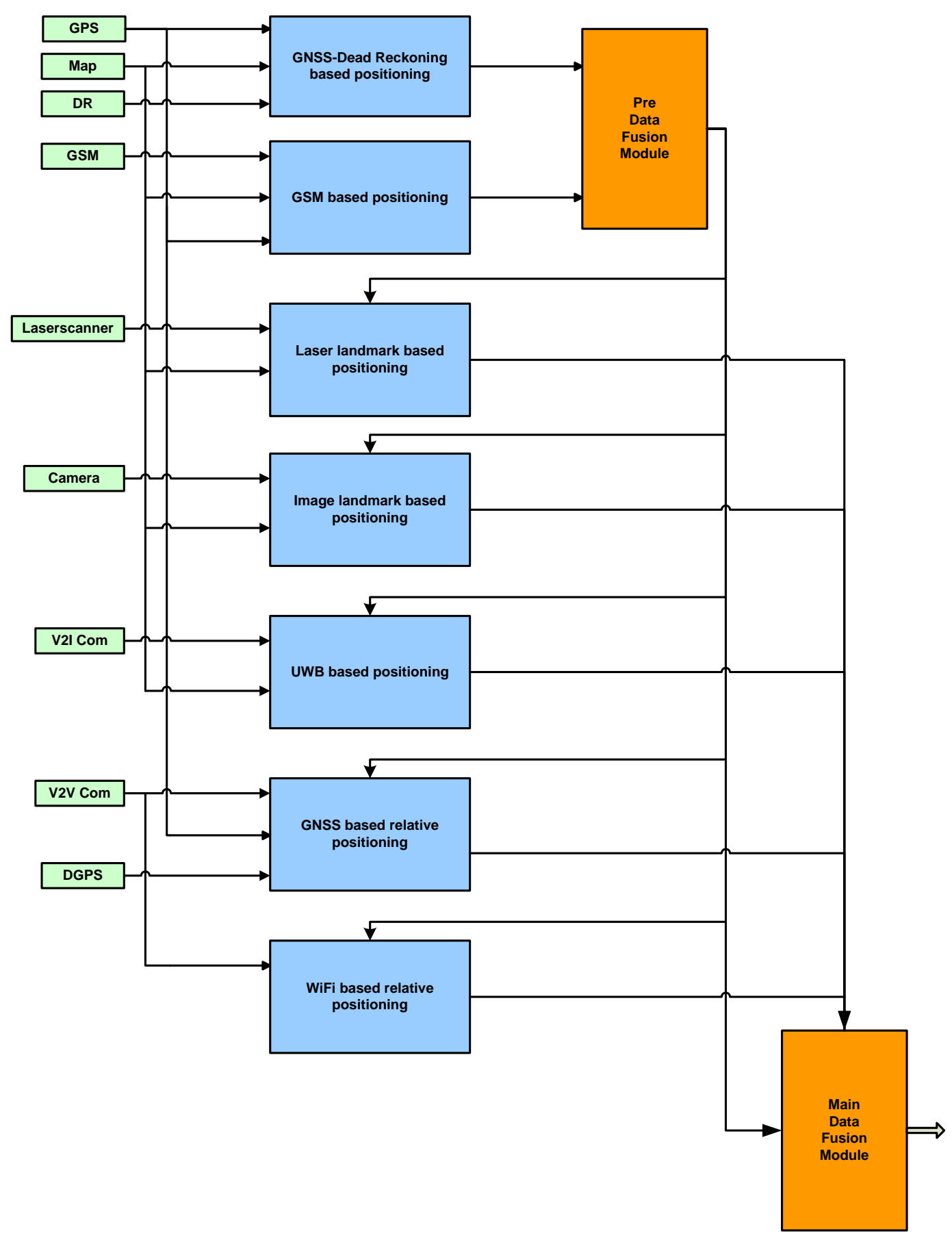

Fig. 1. General structure of the SiNTECH positioning module. Several positioning technologies which have their advantages in different environments are combined using a data fusion module.

case a user's receiver needs at least four satellites in view for a 3D position determination. When using more than four satellites, the position solution is over-determined but this can increase the reliability and the accuracy of the result. The mean accuracy of GPS positions ranges between 10 and 20 meters depending on the satellite constellation but can also reach better values. Galileo is even asserted to reach about 4 meter accuracy with the Open Service.

For an absolute positioning with a higher accuracy in the sub-meter range, a second frequency band and a differential mode with special receiver is needed. A precise located reference receiver station compares the measured pseudoranges with its own position and distributes it's calculated differential correction data to other moving receiver (e.g. via a radio link). With that technology, DGPS services can reach accuracy in the centimetre range but always need a stationary local reference station. Another new approach is the exchange of unprocessed raw measurement data (mainly pseudo-ranges 


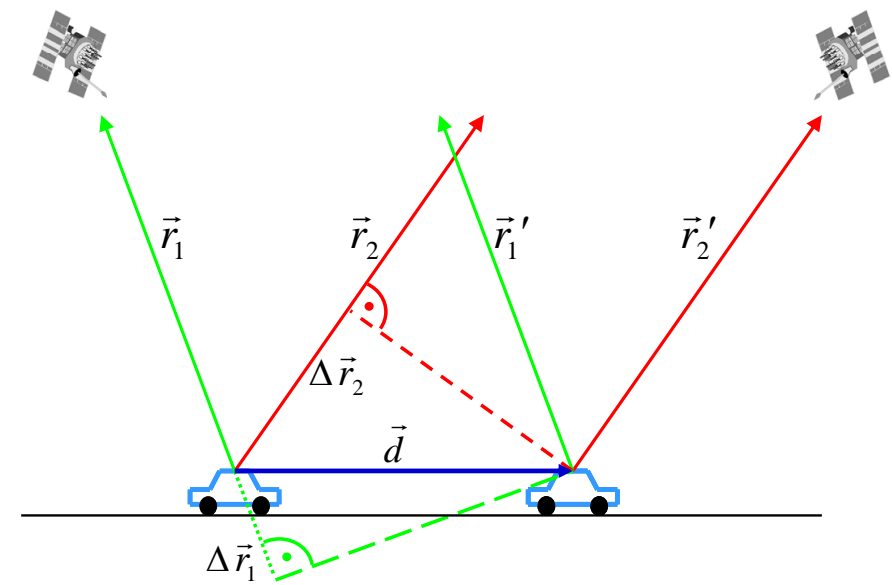

Fig. 2. If two vehicles are close to each other, the difference of their GPSpseudoranges is used to calculate an accurate distance vector between them.

between a vehicle and the received satellites) [2].

If a mobile receiver can detect its position by measuring pseudo-ranges to several GNSS satellites, it can also output the unprocessed pseudo-ranges with a high precise time stamp from the satellite system time. This time reference is the processed result additional to the position, whereby four satellites are needed. If an onboard computer receives data of another receiver in the near vicinity then the pseudo-ranges of both receivers can be compared to each other and a range difference to every satellite can be determined.

Since both signals follow almost the same path and will be influenced by the same effects, the absolute pseudo-range errors through ionospheric signal refraction will not affect the result of the pseudo-range differences used for the calculation of the relative position vector $\vec{d}$, whereas other effects appearing at the surface can result in different reception conditions. These effects can be the shadowing of the signals by buildings, vehicles or vegetation or the reflection from any kind of object. Using signals at different receiver locations (e.g. at two vehicle positions) at the same time from the same satellites, it has to be assured that the conditions for the reception at either location is identical. Only the uninfluenced pseudo ranges can be compared for the difference. If one signal is shadowed or reflected this would cause a large error in the calculation of the pseudo range differences and thus in the relative position result. For that the signals in each receiver have to be analysed with respect to their signal quality (signal strength or the $\mathrm{S} / \mathrm{N}$ ratio and bit error rate). This value has to be considered for each pseudo-range comparison of each vehicle as well to exclude diverse signals from further processing.

To ensure the reception of at least three satellites without signal interferences at several vehicle locations at the same time, the number of visible satellites has to be as high as possible. Thus, more satellites in orbit will lead to a higher probability for the reception of enough satellites on the ground even within critical areas such as urban or mountain areas. Using the additional GNSS-satellites like Galileo or Glonass, the critical threshold of visible navigation satellites required for 2D- (at least three satellites) and even for 3D-navigation (at least four satellites) will almost always be reached regardless whether for the absolute or relative positioning.

Other benefits from using Galileo are already well known within the user community. Galileo offers not only higher availability due to more orbiting satellites but also higher signal quality, positional accuracy, and reliability, as well as service guarantees for Safety-of-Life services (SoL). This can particulary be relevant if absolute or relative positioning technologies will be applied for driver assistance, vehicle or road safety applications as adressed in the SAFESPOT project. The safety and reliability aspect is also closely connected to the integrity monitoring functions for Galileo. The permanently monitored satellite signals enable the creation of information about the signal condition of each satellite and the calculated position resulting from the utilisation of a dedicated signal. When a signal is interrupted or degraded, this information can be provided to any user.

Thus, the utilisation of extended GNSS technology can again support an advanced approach for vehicle safety application but needs also to be combined with other sensors. A final sensor fusion where GNSS is only one minor component is essential for SAFESPOT as well as for other applications.

\section{B. Communication-based Vehicle Positioning}

Another approach is to use Ultra-Wideband (UWB) technology for determining the distance to other vehicles or elements of the road infrastructure, which therefore have to be equipped with active tags. By applying symmetrical double sided two way ranging (SDS-TWR) algorithms, the round trip time (RTT) of the signal and thus the corresponding distance can be measured.

Additionally, two approaches using telecommunication signals are being investigated within the SINTECH subproject: For long range localization, the Broadcast Control Channel (BCCH) of the GSM system can be monitored and used as a backup system if GPS service is not available. Concerning short range localization, particular signal properties of 802.11 (WiFi) and other wireless standards are being studied in order to find useful parameters for position estimation.

Both approaches are mainly based on the properties of the propagation of electromagnetic fields (in particular attenuation and time delay), but also other useful characteristics are being studied.

\section{Landmark-based Positioning I: Image Features}

Positioning using image features as landmarks is currently not state of the art. However, lane tracking systems can be regarded as a supposition to this approach, as they determine the position of lane markings relative to the ego-vehicle. If the exact shape of lane is included into a digital map, the position of the ego-vehicle relative to this map can be determined. Previous work in this field has been done by Cramer et al. [3]. 

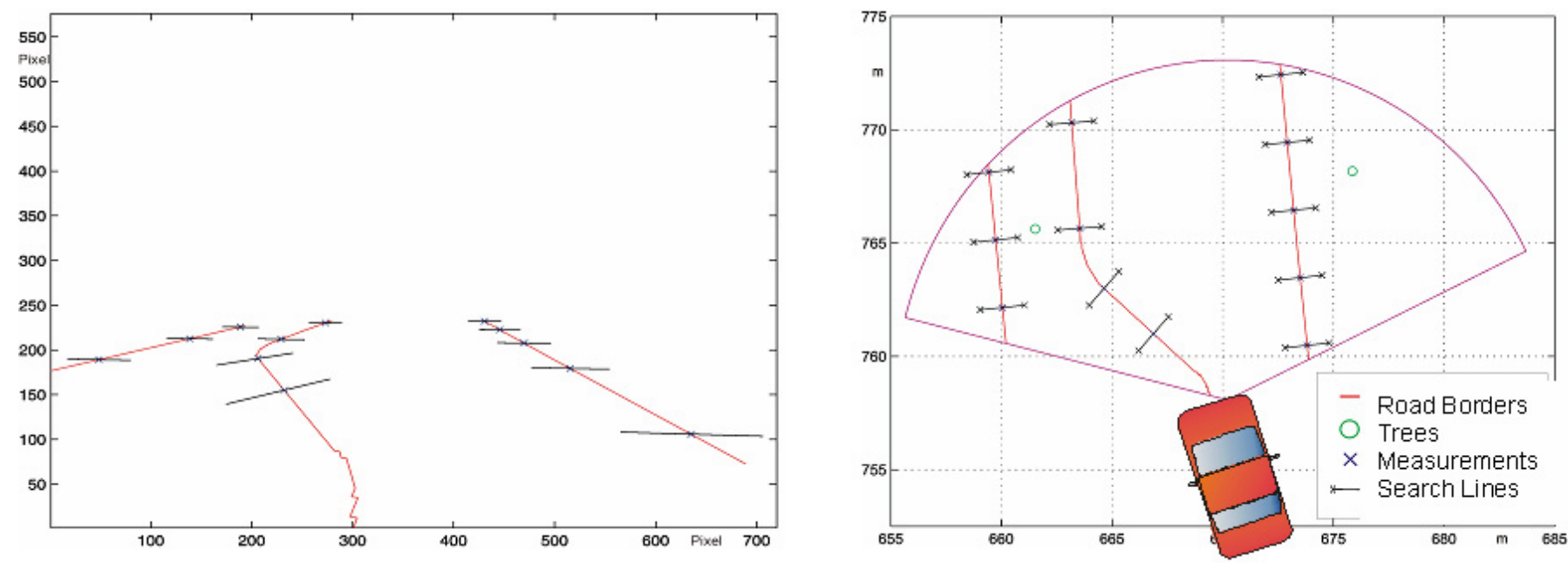

Fig. 3. Transformation of map features into the vehicle coordinate (right). In the next step, the predicted positions of the features in the image plane are calculated (left).

The basic idea of the new approach which is proposed in this paper is to match different lines, which can be extracted from a digital map, with the lines that can be obtained from the image of a vision system. An example for such lines are lane markings, but other lines are also processed. For this approach, two conditions have to be fulfilled:

- The digital map must contain data from which the position and geometrical description of white lines or road borders can be calculated. In SAFESPOT, this is achieved by developing local dynamic maps (LDM) [4]. Those maps contain among others detailed geometric information about the shape of the lane and the position of landmarks in the vicinity of the ego-vehicle. For that, the LDM has to be updated regularly in order to provide present, relevant information.

- The algorithm needs a basic initial position. After starting the system, a first position estimate from a GNSS- or GSM-based module (refer to 1) is used. In later calculation cycles, the position from the last fusion step which has been predicted to the required time value using a dynamic model of the ego vehicle can also be used.

With these conditions being fulfilled, the approach is based on the following processing steps:

1) The initial position is used to search in the map data for image features (lines or road borders), which are in the field of view of the image sensor. These features are extracted from the map and transformed into the vehicle coordinate system using the initial position and heading of the vehicle. This is shown on the right side of figure 3 . The next step is the transformation from the vehicle coordinate system into the image plane. This is shown on the left side of figure 3 .

2) Along these transformed map lines (which can be regarded as predictions), orthogonal search lines are defined. The length of the search lines depends on the uncertainties of the map data, the initial position, and the heading information of the vehicle.

3) Along these search lines, image-processing algorithms are used to calculate the most probable position of the predicted lines. For that the image processing module is using edge detection operations such as Sobel- or Cannyfilters in order to find sudden changes in the grey value of the image. In addition, a hough transformation is used to transform the detected edge points into hough space. By searching the hough space for local maxima, straight lines within the image can be identified. The results of this process are used as measurements for the next step.

4) Finally, an extended Kalman-Filter is used to fuse all measured detections with the predictions. In a previous step, association uncertainties which may occur when there is more than one detection per search line have to be solved. For that, different approaches are possible which may solve the problem using the distance between the measurements (nearest neighbour approach) or the amplitudes of the detections (strongest neighbour approach). However, it shows that a failure in associating the detections correctly may result in a significant disturbance of the result. Thus, the non-neighbour approach is used, which in case of more than one detections per search line deletes every one of them.

The remaining measurements are combined with the predictions by the Extended Kalman Filter (EKF).

\section{Landmark-based Positioning II: Laserscanner Features}

Laser scanners provide another possibility to detect and localize natural landmarks. This functionality has until now mainly been used in intersection environments (e. g. within the InTERSAFE subproject of PRevent) [5]. For that, a grid map of the intersection is generated using a laserscanner. That is, the regarded region is divided into small boxes and the number of measurement points in each box is accumulated. Then, the detections have to be associated to data from a digital map (e. g. using the algorithm described in [6]). 
The new approach used in SAFESPOT extends the current ability to additional environments, such as rural areas or highways. The main challenge in these environments is to achieve sufficient positioning accuracy with a smaller amount of features at higher ego velocities [7]. For that, the set of features used as landmarks is extended to reflection posts, lane markings, crash barriers and kerbstones. All these landmarks will be included in the digital maps which are being used within SAFESPOT.

\section{DATA Fusion For ACCURATE Position Estimation}

One main challenge of the positioning task is to combine the information from different sources in a meaningful way. For that, a data fusion module is being developed. This module has to take several constraints into account. At first, not every localization method is able to deliver its information all the time. For instance, GNSS-based positioning will fail to provide exact results in tunnels, while communication-based positioning (e. g. V2I or V2V) shows its advantages especially in such an environment. Additionally, the accuracy of every single source may change over time, due to the availability of landmarks or communication signals.

In order to find an appropriate data fusion algorithm, several basic approaches are taken into account, which will be briefly explained in the following:

a) Usage of $2^{\text {nd }}$ order statistics (Kalman filter): One possibility is to use statistical descriptions of uncertain data (i. e. mean values and covariance matrices). These information can be combined using a Gauss-Markov estimation approach. Additionally, if an appropriate motion model for the host vehicle is available, a Kalman filter can be used to determine the optimal position estimation.

b) Dempster-Shafer method: Dempster-Shafer theory is a mathematical concept of evidence based on belief functions and plausible reasoning, which is used to combine separate pieces of information to calculate the probability of an event. Dempster-Shafer theory is a generalization of the Bayesian theory of subjective probability, which is based on two ideas: obtaining degrees of belief for one question from subjective probabilities for a related question, and Dempster's rule for combining such degrees of belief when they are based on independent items of evidence.

c) Rule based systems / Fuzzy logic: Not all the localization tasks deliver accurate position estimations under all conditions. Therefore, also a rule based system can help to choose the best localization task. If the decision is assigned with uncertainty, the usage of fuzzy operations is one way to estimate the best position.

\section{EXPECTED RESULTS}

With the cooperative SAFESPOT positioning approach, it will be possible to determine the location of a vehicle with sub-meter accuracy. That is, an application will be able to detect in which lane the vehicle is travelling. By combining this ability with the outcomes of the two other SINTECH fields - local dynamic maps and vehicular ad-hoc networks - it will be possible to develop a safety margin assistant, which provides the driver with concrete recommendations for how to avoid potential dangerous situations.

Another advantage of the SAFESPOT positioning approach is an improved availability, which results from the combination of different information sources. For instance, in scenarios where GPS-signals are not available (e. g. tunnels or urban environments), it will still be possible to localize the vehicle by using one of the alternative approaches.

Furthermore, by using data fusion algorithms it is possible to provide the application with information about the reliability (i. e. uncertainty) of the position estimate. Thus, the application may decide by itself if the delivered accuracy is sufficient and by this means provide its functionality in a more reliable way.

\section{REFERENCES}

[1] R. Brignolo, "Co-operative Road Safety - The SAFESPOT Integrated Project," in APSN - APROSYS Conference, May 2006. [Online]. Available: http://www.safespot-eu.org/pages/docs/ SF_Presentation_ASFN_APROSYS_CONF_Vienna.pdf

[2] M. Schlingelhof, R. Kuehne, and D. Krajzewicz, "New GNSSBased Approaches For Advanced Driver Assistance Systems," in TRB 2006 (85th Annual Meeting), January 2006. [Online]. Available: http://elib.dlr.de/21758

[3] H. Cramer, U. Scheunert, and G. Wanielik, "A New Approach for Tracking Lanes by Fusing Image Measurements with Map Data," in Proceedings of IEEE Intelligent Vehicle Symposium, Parma, Italy, 2004.

[4] C. Barthels, "Local dynamic maps for cooperative systems," in ITS World Congress, October 2006.

[5] K. Fuerstenberg and T. Weiss, "Feature-level map building and object recognition for intersection safety applications," in Intelligent Vehicles Symposium, 2005. Proceedings. IEEE, 6-8 June 2005, pp. 490-495.

[6] T. Weiss, N. Kaempchen, and K. Dietmayer, "Precise ego-localization in urban areas using Laserscanner and high accuracy feature maps," in Intelligent Vehicles Symposium, 2005. Proceedings. IEEE, 6-8 June 2005, pp. 284-289.

[7] A. Brakemeier et al., "D3.2.1 - Technical Scenarios," 2006, Deliverable of the SAFESPOT Integrated Project. [Online]. Available: http://www. safespot-eu.org/pages/docs/D3.2.1_Technical_Scenarios_Summary.pdf 